Report no. 04/21

\title{
Finite Element Solution of A Membrane Covered Electrode Problem
}

\author{
Kathryn Harriman, David Gavaghan and Endre Süli
}

\begin{abstract}
Membrane covered oxygen sensors, or Clark electrodes, are used for monitoring the concentration of oxygen in blood. The operation of such sensors is governed by the diffusion equation with different diffusion coefficients in different sub-domains. The form of the boundary conditions and the material interface conditions means that the derivative of the solution has discontinuities which restrict the convergence of standard numerical methods on regular meshes. We describe and computationally compare adaptive finite element methods based on continuous and discontinuous basis functions to overcome this problem.
\end{abstract}

Key words and phrases: Finite element methods, discontinuous Galerkin methods, linear functionals, membrane covered electrode

Oxford University Computing Laboratory

Numerical Analysis Group

Wolfson Building

Parks Road

Oxford, England OX1 3QD

July, 2004 


\section{Introduction}

The Clark electrode was first developed in 1956 by Leland Clark [1] as a means of measuring blood oxygen tension $\left(P_{\mathrm{O}_{2}}\right)$. Before this the most reliable indication of a patient's oxygen level had been the colour of their skin. The Clark electrode is still the main means of monitoring blood $P_{\mathrm{O}_{2}}$ in clinical medicine and is now used in several other areas of clinical research including transcutaneous oxygen monitoring in babies, haemoglobin dissociation and molecular genetics. Applications have also spread outside clinical medicine and now include sewage treatment, soil chemistry and the production of wine and beer.

A schematic of a Clark oxygen sensor is shown in Figure 1. The cathode is made from an unreactive noble metal, for example platinum or gold. The anode is generally also made from an unreactive noble metal such as silver. An external voltage is applied for reduction to take place. The electrode assembly is then immersed in aqueous electrolyte solution and protected from poisoning by a tightly stretched plastic membrane which is permeable to oxygen.

The simplest form of the chemical reaction which takes place at the cathode when oxygen is reduced is

$$
\mathrm{O}_{2}+2 \mathrm{H}_{2} \mathrm{O}+4 e^{-}=4 \mathrm{OH}^{-} \text {. }
$$

This sets up a concentration gradient of oxygen within the electrolyte allowing oxygen to diffuse towards the cathode. We assume that the potential difference between the two electrodes is sufficiently large that all oxygen reaching the cathode is reduced. Hence we obtain a current which is directly proportional to the oxygen concentration.

\section{Mathematical Model}

Oxygen sensors are used in practice with a suitably negative potential to give diffusionlimited current and so we may assume that all oxygen molecules reaching the cathode are reduced. The resulting concentration gradient allows the transport of oxygen to the cathode to be modelled using the diffusion equation. We model the sensor by assuming that the cathode is a disc shaped electrode of radius $r_{c}$ (typically ranging from $10 \mu \mathrm{m}$ to $100 \mu \mathrm{m})$ embedded in an infinite planar insulating material. This gives symmetry about the $z$-axis so that we may reduce the problem to one in 2-dimensional cylindrical polar coordinates as shown in Figure 2. Above the cathode we have three layers:

1. an electrolyte layer $(e)$ of thickness $z_{e}$ (typically $z_{e}$ ranges between $2 \mu \mathrm{m}$ and $10 \mu \mathrm{m}$ );

2. a membrane layer $(m)$ of thickness $z_{m}$ (typically $z_{m}$ ranges between $5 \mu \mathrm{m}$ and $25 \mu \mathrm{m})$. The membrane is porous to oxygen but typically has a diffusion coefficient at least an order of magnitude lower than that of the electrolyte;

3. a layer of sample $(s)$ taken to be infinitely thick. The diffusion coefficient is the same order of magnitude as that of the electrolyte. 


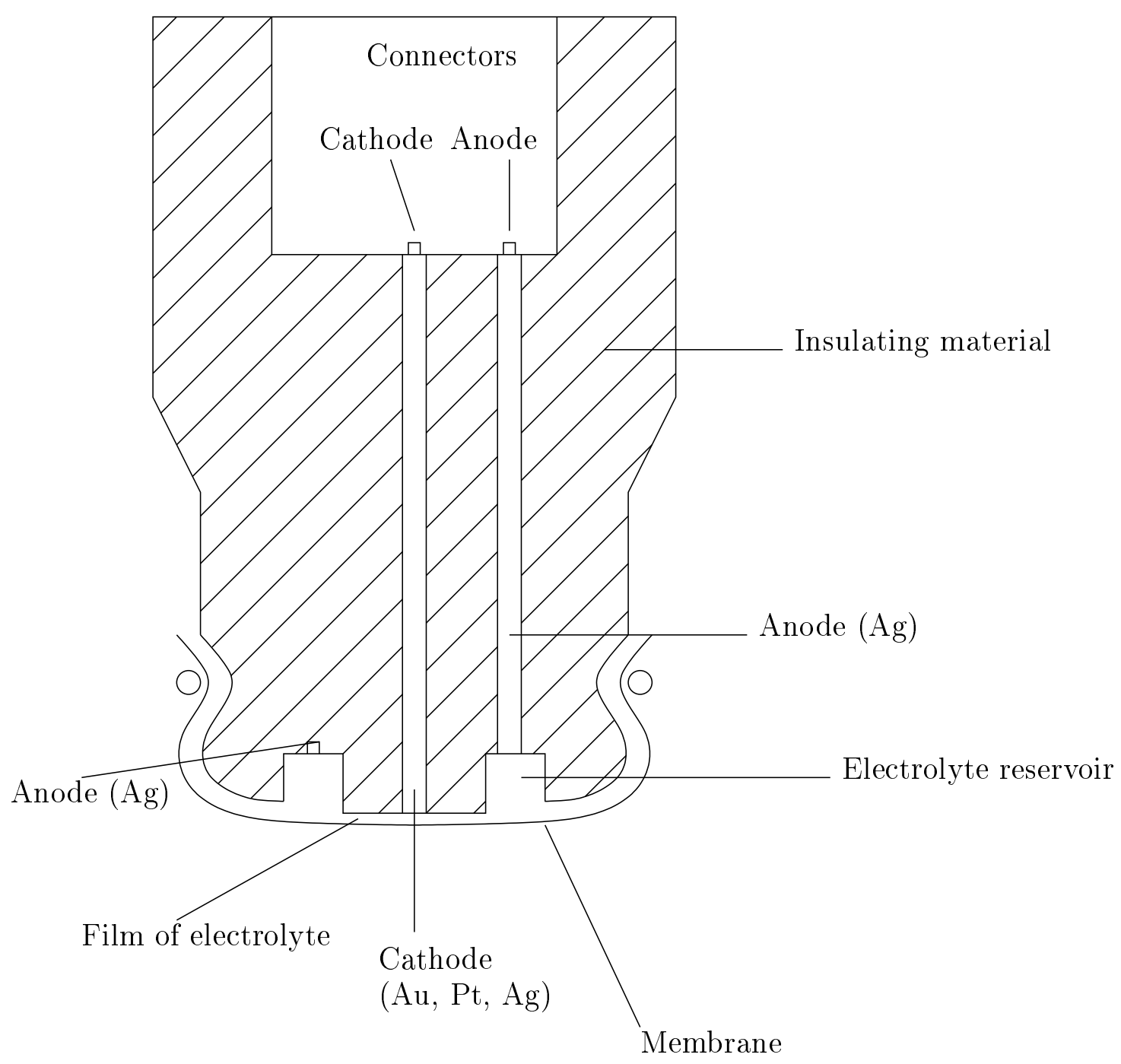

Figure 1: The Clark electrode.

The partial pressure of oxygen $p$ is related to the concentration of oxygen $c$ by Henry's Law: $c=\alpha_{k} p$ where $k=e, m, s$ and $\alpha_{k}$ is the solubility in the given layer. Thus the partial pressure also obeys the diffusion equation

$$
P_{k}\left(\frac{1}{r} \frac{\partial}{\partial r}\left(r \frac{\partial p}{\partial r}\right)+\frac{\partial^{2} p}{\partial z^{2}}\right)=0,
$$

in each layer where $P_{k}$ is the relevant permeability related to the diffusion coefficient by $P_{k}=\alpha_{k} D_{k}$. Additionally the partial pressure has the advantage that it is continuous across the interfaces between layers whilst the concentration is not. The current is then given by

$$
I=2 \pi n F P_{e} \int_{0}^{r_{c}}\left(\frac{\partial p}{\partial z}\right)_{z=0} r \mathrm{~d} r
$$




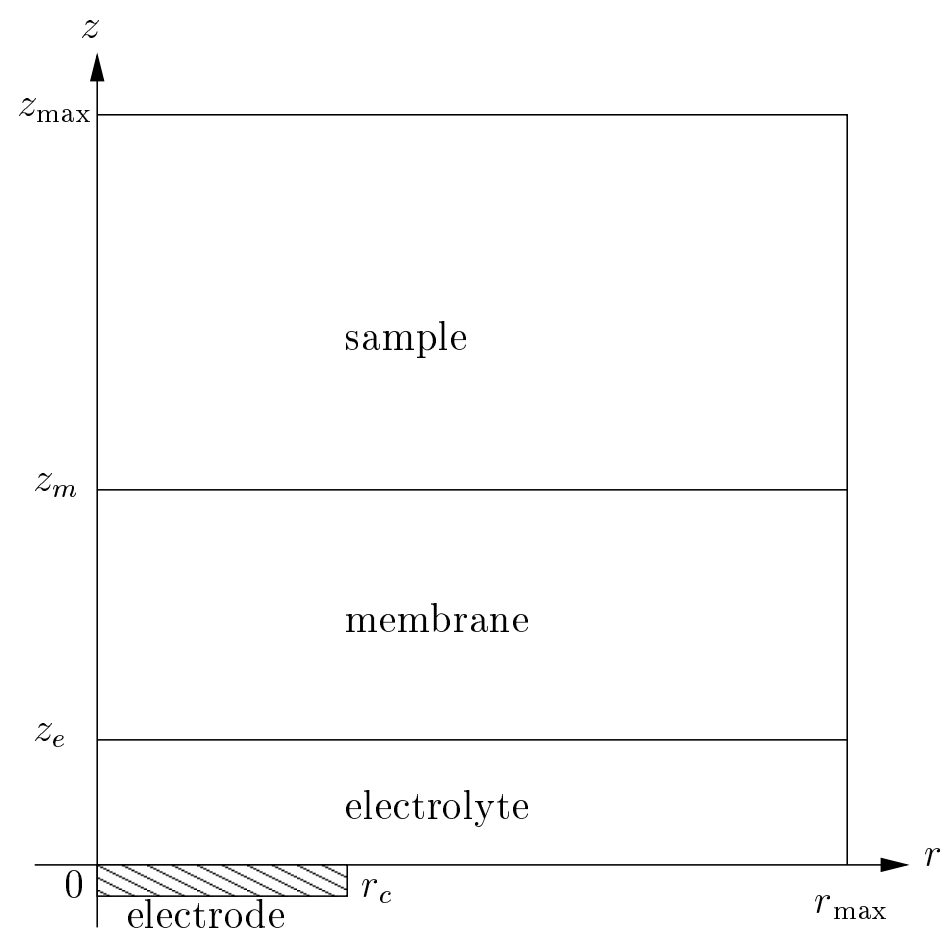

Figure 2: The geometry for the mathematical model of the Clark electrode.

where $F$ is the Faraday constant and $n$ is the number of electrons per molecule transferred in the reduction reaction. Since we are assuming that the reaction (1.1) takes place we shall take $n=4$. We assume that oxygen depletion takes place in all three layers but also that there exist distances $r_{\max }$ and $z_{\max }$ at which no further oxygen depletion takes place so that the problem may be solved in the finite domain $\left[0, r_{\max }\right] \times\left[0, z_{\max }\right]$.

\subsection{Boundary and Internal Conditions}

We shall solve for the dimensionless partial pressure $p^{\prime}=p / p_{0}$ where $p_{0}$ is the bulk partial pressure. We also normalise the spatial coordinates with respect to the electrode radius (i.e. set $\hat{r}=r / r_{c}$ and $\hat{z}=z / r_{c}$ and drop hats). Since we assume that all oxygen molecules which reach the cathode are reduced we have the boundary condition

$$
p^{\prime}=0 \quad \text { on } z=0,0 \leq r \leq 1 .
$$

Since no oxygen can escape through the insulating surface we have

$$
\frac{\partial p^{\prime}}{\partial z}=0 \quad \text { on } z=0,1<r<r_{\max }
$$

The symmetry of the problem requires the boundary condition

$$
\frac{\partial p^{\prime}}{\partial r}=0 \quad \text { on } r=0,0<z<z_{\max }
$$


to be imposed. The set of boundary conditions is completed by imposing that the partial pressure at infinity should equal the bulk partial pressure, or, on our bounded domain and in dimensionless variables, we require

$$
p^{\prime}=1 \quad \text { on } r=r_{\max } \text { and } z=z_{\max } .
$$

We also have internal conditions to be satisfied at the material interfaces. We require the partial pressure to be continuous and so

$$
\begin{aligned}
& p_{z=z_{e}^{-}}^{\prime}=p_{z=z_{e}^{+}}^{\prime}, \\
& p_{z=z_{m}^{-}}^{\prime}=p_{z=z_{m}^{+}}^{\prime} .
\end{aligned}
$$

We also require the oxygen fluxes to be continuous across the material interfaces and so our mathematical model is completed by imposing the conditions

$$
\begin{gathered}
P_{e}\left(\frac{\partial p^{\prime}}{\partial z}\right)_{z=z_{e}^{-}}=P_{m}\left(\frac{\partial p^{\prime}}{\partial z}\right)_{z=z_{e}^{+}}, \\
P_{m}\left(\frac{\partial p^{\prime}}{\partial z}\right)_{z=z_{m}^{-}}=P_{s}\left(\frac{\partial p^{\prime}}{\partial z}\right)_{z=z_{m}^{+}} .
\end{gathered}
$$

We note that this final requirement results in discontinuities in $\partial p^{\prime} / \partial z$ across the material interfaces.

The dimensionless current is now given by

$$
I^{\prime}=\frac{\pi}{2} \int_{0}^{1}\left(\frac{\partial p^{\prime}}{\partial z}\right)_{z=0} r \mathrm{~d} r .
$$

\subsection{Analytical Expression for the Current}

Several analytical expressions for the current have been given by Galcercan et al. [2] . They introduce the dimensionless parameters

$$
\begin{aligned}
\epsilon_{1} & =\frac{P_{e}-P_{m}}{P_{e}+P_{m}}=\frac{P_{e} / P_{m}-1}{P_{e} / P_{m}+1}, \\
\epsilon_{2} & =\frac{P_{s}-P_{m}}{P_{s}+P_{m}}=\frac{P_{s} / P_{m}-1}{P_{s} / P_{m}+1},
\end{aligned}
$$

which depend on the ratios of the permeabilities. We note that these expressions give

$$
\begin{aligned}
& \frac{P_{e}}{P_{m}}=\frac{1+\epsilon_{1}}{1-\epsilon_{1}}, \\
& \frac{P_{s}}{P_{m}}=\frac{1+\epsilon_{2}}{1-\epsilon_{2}} .
\end{aligned}
$$

They then define the function $f_{3}(\cdot)$ by

$$
f_{3}(\lambda)=\frac{\epsilon_{1} e^{-2 \lambda z_{e}}-\epsilon_{2} e^{-2 \lambda z_{m}}}{1-\epsilon_{1} \epsilon_{2} e^{2 \lambda\left(z_{e}-z_{m}\right)}-\epsilon_{1} e^{-2 \lambda z_{e}}+\epsilon_{2} e^{-2 \lambda z_{m}}},
$$


and constants $L_{m, n}$ by

$$
L_{m, n}=(4 n+1) \int_{0}^{\infty} \frac{2}{\lambda} f_{3}(\lambda) J_{2 m+1 / 2}(\lambda) J_{2 n+1 / 2}(\lambda) \mathrm{d} \lambda,
$$

where $J_{\nu}(\cdot)$ is the Bessel function of order $\nu$. Then, given the infinite linear system

$$
\begin{aligned}
a_{0}+\sum_{m=0}^{\infty} L_{m, 0} a_{m} & =\sqrt{\frac{2}{\pi}}, \\
a_{n}+\sum_{m=0}^{\infty} L_{m, n} a_{m} & =0, \quad n>0,
\end{aligned}
$$

the exact current is given by

$$
I^{\prime}=\sqrt{\frac{\pi}{2}} a_{0} .
$$

It should be noted that although (2.18) represents the exact value of the steady state current, in practice it only determines an approximate (although very accurate) current since the infinite series must be truncated and the integrals defining the constants $L_{m, n}$ must be approximated over a finite range of integration. More details of the precise evaluation of (2.18) are given in [2].

\subsection{Approximate Analytical Expression for the Current}

Galceran et al. [2] also give an approximate analytical expression for the current valid for membranes which are not too thick and not too impermeable (i.e. for parameter regimes in which neither $z_{m} \gg z_{e}$ nor $\epsilon_{1}, \epsilon_{2} \rightarrow 1$ hold). The approximate analytical expression is given by

$$
\begin{aligned}
I^{\prime}= & \left(1+2\left[\epsilon_{1} j_{0,0}\left(2 z_{e}\right)-\epsilon_{2} j_{0,0}\left(2 z_{m}\right)+\epsilon_{1} k_{1} e^{2 k_{2} \lambda_{1}} j_{0,0}\left(2\left(z_{e}+k_{2}\right)\right)\right.\right. \\
& \left.\left.-\epsilon_{2} k_{1} e^{2 k_{2} \lambda_{1}} j_{0,0}\left(2\left(z_{m}+k_{2}\right)\right)\right]\right)^{-1},
\end{aligned}
$$

where

$$
\begin{aligned}
\lambda_{1} & =\frac{1}{2\left(z_{m}-z_{e}\right)} \ln \left(\frac{\epsilon_{2} z_{m}}{\epsilon_{1} z_{e}}\right) \\
\lambda_{2} & =\frac{1}{2\left(z_{m}-z_{e}\right)} \ln \left(\frac{\epsilon_{2} z_{m}^{2}}{\epsilon_{1} z_{e}^{2}}\right) \\
d(\lambda) & =1-\epsilon_{1} \epsilon_{2} e^{2 \lambda\left(z_{e}-z_{m}\right)}-\epsilon_{1} e^{-2 \lambda z_{e}}+\epsilon_{2} e^{-2 \lambda z_{m}} \\
k_{1} & =\frac{1}{d\left(\lambda_{1}\right)}-1 \\
k_{2} & =\frac{1}{2\left(\lambda_{2}-\lambda_{1}\right)} \ln \left(\frac{k_{1}}{\frac{1}{d\left(\lambda_{2}\right)}-1}\right) \\
j_{0,0}(x) & =\frac{2}{\pi} \tan ^{-1}\left(\frac{2}{x}\right)-\frac{x}{2 \pi} \ln \left(1+\frac{4}{x^{2}}\right) .
\end{aligned}
$$




\section{Continuous Finite Element Solution}

We let $\Omega=\left(0, r_{\max }\right) \times\left(0, z_{\max }\right)$ and we let $\Omega_{e}, \Omega_{m}$ and $\Omega_{s}$ represent the domains corresponding to electrolyte, membrane and sample respectively with boundaries $\partial \Omega_{e}$, $\partial \Omega_{m}$ and $\partial \Omega_{s}$. We define the trial and test spaces $H_{E}^{1}(\Omega)$ and $H_{E_{0}}^{1}(\Omega)$ by

$$
\begin{aligned}
& H_{E}^{1}(\Omega)=\left\{v \in H^{1}(\Omega): v \text { satisfies the Dirichlet boundary conditions }\right\} \\
& H_{E_{0}}^{1}(\Omega)=\left\{v \in H^{1}(\Omega): v=0 \text { on the Dirichlet boundary }\right\} .
\end{aligned}
$$

Thus we have, for any $v \in H_{E_{0}}^{1}(\Omega)$,

$$
P_{e} \int_{\Omega_{e}}-\nabla^{2} p^{\prime} v \mathrm{~d} \Omega+P_{m} \int_{\Omega_{m}}-\nabla^{2} p^{\prime} v \mathrm{~d} \Omega+P_{s} \int_{\Omega_{s}}-\nabla^{2} p^{\prime} v \mathrm{~d} \Omega=0
$$

Applying Green's theorem gives

$$
\begin{aligned}
P_{e} \int_{\Omega_{e}} \nabla p^{\prime} \cdot \nabla v \mathrm{~d} \Omega+P_{m} \int_{\Omega_{m}} \nabla p^{\prime} \cdot \nabla v \mathrm{~d} \Omega+P_{s} \int_{\Omega_{s}} \nabla p^{\prime} \cdot \nabla v \mathrm{~d} \Omega & \\
- & P_{e} \int_{\partial \Omega_{e}} v \frac{\partial p^{\prime}}{\partial n} \mathrm{~d} s-P_{m} \int_{\partial \Omega_{m}} v \frac{\partial p^{\prime}}{\partial n} \mathrm{~d} s-P_{s} \int_{\partial \Omega_{s}} v \frac{\partial p^{\prime}}{\partial n} \mathrm{~d} s=0 .
\end{aligned}
$$

Noting that $v=0$ on the Dirichlet parts of the boundary and that $\partial p^{\prime} / \partial n=0$ on the remainder of the boundary we see that

$$
\begin{aligned}
P_{e} \int_{\Omega_{e}} \nabla p^{\prime} \cdot \nabla v \mathrm{~d} \Omega+P_{m} \int_{\Omega_{m}} \nabla p^{\prime} \cdot \nabla v \mathrm{~d} \Omega+P_{s} \int_{\Omega_{s}} \nabla p^{\prime} \cdot \nabla v \mathrm{~d} \Omega & \\
& -\int_{0}^{r_{\max }} v\left(P_{e}\left(\frac{\partial p^{\prime}}{\partial z}\right)_{z=z_{e}^{-}}-P_{m}\left(\frac{\partial p^{\prime}}{\partial z}\right)_{z=z_{e}^{+}}\right) r \mathrm{~d} r \\
& -\int_{0}^{r_{\max }} v\left(P_{m}\left(\frac{\partial p^{\prime}}{\partial z}\right)_{z=z_{m}^{-}}-P_{s}\left(\frac{\partial p^{\prime}}{\partial z}\right)_{z=z_{m}^{+}}\right) r \mathrm{~d} r=0 .
\end{aligned}
$$

Applying the equations (2.8) which impose continuity of the flux across the material interfaces and dividing by $P_{m}$ we see that the weak formulation of the problem is to find $p^{\prime} \in H_{E}^{1}(\Omega)$ such that

$$
\int_{\Omega_{e}} \frac{P_{e}}{P_{m}} \nabla p^{\prime} \cdot \nabla v \mathrm{~d} \Omega+\int_{\Omega_{m}} \nabla p^{\prime} \cdot \nabla v \mathrm{~d} \Omega+\int_{\Omega_{s}} \frac{P_{s}}{P_{m}} \nabla p^{\prime} \cdot \nabla v \mathrm{~d} \Omega=0 \quad \forall v \in H_{E_{0}}^{1}(\Omega) .
$$

\subsection{Expression for the Current}

We define the function $\psi$ on the Dirichlet boundary by

$$
\psi= \begin{cases}1 & z=0,0 \leq r \leq 1 \\ 0 & r=r_{\max } \text { or } z=z_{\max }\end{cases}
$$


We then define the Sobolev space $H_{\psi}^{1}(\Omega)$ by

$$
H_{\psi}^{1}(\Omega)=\left\{v \in H^{1}(\Omega): v=\psi \text { on the Dirichlet boundary }\right\} .
$$

Now we have, as before,

$$
P_{e} \int_{\Omega_{e}}-\nabla^{2} p^{\prime} v \mathrm{~d} \Omega+P_{m} \int_{\Omega_{m}}-\nabla^{2} p^{\prime} v \mathrm{~d} \Omega+P_{s} \int_{\Omega_{s}}-\nabla^{2} p^{\prime} v \mathrm{~d} \Omega=0 .
$$

Applying Green's theorem, using the boundary conditions for $p^{\prime}$ on the Neumann boundary, for $v$ on the Dirichlet boundary and using the material interface conditions (2.8) gives

$$
\begin{aligned}
P_{e} \int_{\Omega_{e}} \nabla p^{\prime} \cdot \nabla v \mathrm{~d} \Omega+P_{m} \int_{\Omega_{m}} \nabla p^{\prime} \cdot \nabla v \mathrm{~d} \Omega & +P_{s} \int_{\Omega_{s}} \nabla p^{\prime} \cdot \nabla v \mathrm{~d} \Omega \\
& +\int_{0}^{1} P_{e}\left(\frac{\partial p^{\prime}}{\partial z}\right)_{z=0} r \mathrm{~d} r=0 .
\end{aligned}
$$

Rearranging gives an equivalent definition for the current

$$
\begin{aligned}
I^{\prime}=\frac{\pi}{2} \int_{0}^{1}\left(\frac{\partial p^{\prime}}{\partial z}\right)_{z=0} r \mathrm{~d} r= & -\frac{\pi}{2} \int_{\Omega_{e}} \nabla p^{\prime} \cdot \nabla v \mathrm{~d} \Omega-\frac{\pi}{2} \int_{\Omega_{m}} \frac{P_{m}}{P_{e}} \nabla p^{\prime} \cdot \nabla v \mathrm{~d} \Omega \\
& -\frac{\pi}{2} \int_{\Omega_{s}} \frac{P_{s}}{P_{e}} \nabla p^{\prime} \cdot \nabla v \mathrm{~d} \Omega \\
= & B\left(p^{\prime}, v\right),
\end{aligned}
$$

for all $v \in H_{\psi}^{1}(\Omega)$. We note that $P_{s} / P_{e}=\left(P_{s} / P_{m}\right) \times\left(P_{m} / P_{e}\right)$ and so all coefficients may be expressed in terms of the dimensionless parameters $\epsilon_{1}$ and $\epsilon_{2}$.

\subsection{An A Posteriori Error Bound}

Here we derive an a posteriori bound on the error in the current computed using a piecewise linear finite element approximation to $p^{\prime}$. We define $S^{h}$ to be the subspace of $H^{1}(\Omega)$ consisting of piecewise linear polynomials on a given triangulation $\{\kappa\}$ of $\Omega$ and we define analogous subspaces $S_{E}^{h}, S_{E_{0}}^{h}$ and $S_{\psi}^{h}$ of $H_{E}^{1}(\Omega), H_{E_{0}}^{1}(\Omega)$ and $H_{\psi}^{1}(\Omega)$, respectively. The weak formulation of the problem is thus: find $p^{\prime} \in H_{E}^{1}(\Omega)$ such that

$$
\frac{P_{e}}{P_{m}} B\left(p^{\prime}, v\right)=0 \quad \forall v \in H_{E_{0}}^{1}(\Omega)
$$

The corresponding finite element problem is: find $p_{h}^{\prime} \in S_{E}^{h}$ such that

$$
\frac{P_{e}}{P_{m}} B\left(p_{h}^{\prime}, v_{h}\right)=0 \quad \forall v_{h} \in S_{E_{0}}^{h} .
$$

We then have the definition

$$
I^{\prime}=B\left(p^{\prime}, v\right) \quad \forall v \in H_{\psi}^{1}(\Omega),
$$


for the current and we may approximate the current using

$$
I_{h}^{\prime}=B\left(p_{h}^{\prime}, v_{h}\right) \quad \forall v_{h} \in S_{\psi}^{h} .
$$

We now wish to find a computable upper bound on $\left|I^{\prime}-I_{h}^{\prime}\right|$. To this end we define the dual problem: find $w \in H_{\psi}^{1}(\Omega)$ such that

$$
\frac{P_{e}}{P_{m}} B(\phi, w)=0 \quad \forall \phi \in H_{E_{0}}^{1}(\Omega) .
$$

We now have

$$
\begin{aligned}
I^{\prime}-I_{h}^{\prime} & =B\left(p^{\prime}, v\right)-B\left(p_{h}^{\prime}, v_{h}\right) \\
& =B\left(p^{\prime}, v_{h}\right)-B\left(p_{h}^{\prime}, v_{h}\right) \\
& =B\left(p^{\prime}-p_{h}^{\prime}, v_{h}\right)
\end{aligned}
$$

for any $v_{h} \in S_{\psi}^{h}$ since $H_{\psi}^{1}(\Omega) \subset S_{\psi}^{h}$ and using the bilinearity of $B(\cdot, \cdot)$. Now we take $\phi=p^{\prime}-p_{h}^{\prime} \in H_{E_{0}}^{1}(\Omega)$ in the definition of the dual (3.17) to get

$$
\frac{P_{e}}{P_{m}} B\left(p^{\prime}-p_{h}^{\prime}, w\right)=0
$$

Subtracting (3.21) from (3.20) gives

$$
\begin{aligned}
I^{\prime}-I_{h}^{\prime} & =B\left(p^{\prime}-p_{h}^{\prime}, v_{h}-w\right) \\
& =B\left(p^{\prime}, v_{h}-w\right)-B\left(p_{h}^{\prime}, v_{h}-w\right) \\
& =-B\left(p_{h}^{\prime}, v_{h}-w\right)
\end{aligned}
$$

since taking $v=v_{h}-w$ in (3.13) shows us that $B\left(p^{\prime}, v_{h}-w\right)=0$. Thus we have

$$
\begin{aligned}
I^{\prime}-I_{h}^{\prime}= & \frac{\pi}{2}\left(\int_{\Omega_{e}} \nabla p_{h}^{\prime} \cdot \nabla\left(v_{h}-w\right) \mathrm{d} \Omega+\int_{\Omega_{m}} \frac{P_{m}}{P_{e}} \nabla p_{h}^{\prime} \cdot \nabla\left(v_{h}-w\right) \mathrm{d} \Omega\right. \\
& \left.+\int_{\Omega_{s}} \frac{P_{s}}{P_{e}} \nabla p_{h}^{\prime} \cdot \nabla\left(v_{h}-w\right) \mathrm{d} \Omega\right) \\
= & \frac{\pi}{2}\left(\sum_{\kappa_{e}} \int_{\partial \kappa_{e}} \frac{\partial p_{h}^{\prime}}{\partial n}\left(v_{h}-w\right) \mathrm{d} s+\frac{P_{m}}{P_{e}} \sum_{\kappa_{m}} \int_{\partial \kappa_{m}} \frac{\partial p_{h}^{\prime}}{\partial n}\left(v_{h}-w\right) \mathrm{d} s\right. \\
& \left.+\frac{P_{s}}{P_{e}} \sum_{\kappa_{s}} \int_{\partial \kappa_{s}} \frac{\partial p_{h}^{\prime}}{\partial n}\left(v_{h}-w\right) \mathrm{d} s\right),
\end{aligned}
$$

applying Green's theorem and noting that for piecewise linear finite elements we have $\nabla^{2} p_{h}^{\prime}=0$ in each element of the triangulation. After a little algebra and application of 
the Cauchy-Schwarz inequality we have the final a posteriori error bound

$$
\begin{aligned}
\left|I^{\prime}-I_{h}^{\prime}\right| \leq & \frac{\pi}{4}\left(\sum_{\kappa_{e}}\left\|\left[\frac{\partial p_{h}^{\prime}}{\partial n}\right]\right\|_{L_{2}\left(\partial \kappa_{e}\right)}\left\|v_{h}-w\right\|_{L_{2}\left(\partial \kappa_{e}\right)}\right. \\
& +\frac{P_{m}}{P_{e}} \sum_{\kappa_{m}}\left\|\left[\frac{\partial p_{h}^{\prime}}{\partial n}\right]\right\|_{L_{2}\left(\partial \kappa_{m}\right)}\left\|v_{h}-w\right\|_{L_{2}\left(\partial \kappa_{m}\right)} \\
& \left.+\frac{P_{s}}{P_{e}} \sum_{\kappa_{s}}\left\|\left[\frac{\partial p_{h}^{\prime}}{\partial n}\right]\right\|_{L_{2}\left(\partial \kappa_{s}\right)}\left\|v_{h}-w\right\|_{L_{2}\left(\partial \kappa_{s}\right)}\right),
\end{aligned}
$$

for all $v_{h} \in S_{\psi}^{h}$.

\subsection{Numerical Examples}

First we illustrate the effect of changing the dimensionless parameters $\epsilon_{1}$ and $\epsilon_{2}$. In Figure 3(a) we have taken $z_{e}=0.5$ and $z_{m}=1$; then we have taken $\epsilon_{1}=\epsilon_{2}=0.9214$. This corresponds to $P_{e} / P_{m}=P_{s} / P_{m}=24.45$ meaning that $P_{e}$ and $P_{s}$ are an order of magnitude larger than $P_{m}$. The change in the gradient of the normalised partial pressure can be clearly seen at the material interfaces. In Figure $3(\mathrm{~b})$ we have taken $\epsilon_{1}=\epsilon_{2}=0.1$, meaning $P_{e} / P_{m}=P_{s} / P_{m}=1.22$ so that $P_{e}, P_{m}$ and $P_{s}$ are all of the same order of magnitude. In this case it is much harder to see the change in gradient across the material interfaces.

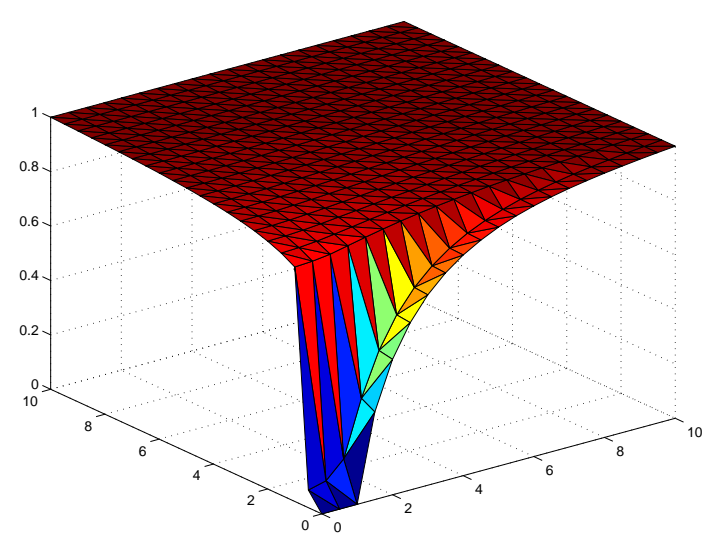

(a) Normalised partial pressure: $z_{e}=0.5$, $z_{m}=1, \epsilon_{1}=\epsilon_{2}=0.9214$

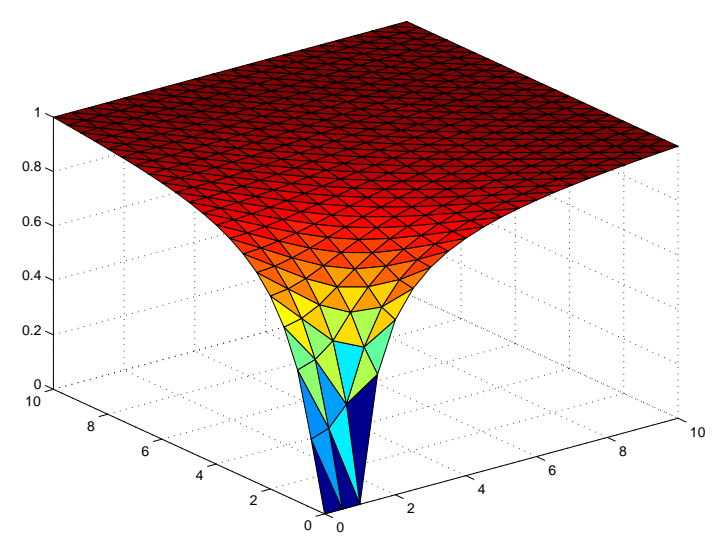

(b) Normalised partial pressure: $z_{e}=0.5$, $z_{m}=1, \epsilon_{1}=\epsilon_{2}=0.1$

Figure 3: Typical solutions for the normalised partial pressure.

Next we compare the current values obtained by our adaptive finite element method with those given by the analytical solution (2.18) and the approximate analytical solution (2.19). In each case we have chosen the adaptive tolerance to be $5 \%$ of the exact current value. The results are shown in Table 1 and we see good agreement in all cases. The 
computations typically required about 10000 nodes and took about one minute of CPU time.

\begin{tabular}{lllcccc}
\hline$z_{e}$ & $z_{m}$ & $\epsilon_{1}=\epsilon_{2}$ & Eq. (2.18) & Eq. (2.19) & $I_{h}^{\prime}$ & error bound \\
\hline 0.5 & 1 & 0.9214 & 0.5141 & 0.5236 & 0.5274 & $2.3302 \times 10^{-2}$ \\
0.5 & 1 & 0.1 & 0.9665 & 0.9663 & 1.0141 & $4.6384 \times 10^{-2}$ \\
2 & 4 & 0.9765 & 0.6672 & 0.7116 & 0.6749 & $3.0289 \times 10^{-2}$ \\
2 & 4 & 0.9214 & 0.7530 & 0.7605 & 0.7580 & $3.4287 \times 10^{-2}$ \\
2 & 4 & 0.2090 & 0.9683 & 0.9681 & 0.9940 & $4.9358 \times 10^{-2}$ \\
2 & 2.5 & 0.9 & 0.8667 & 0.8667 & 0.8811 & $2.8724 \times 10^{-2}$ \\
2 & 2.5 & 0.1 & 0.9942 & 0.9941 & 1.0171 & $4.2267 \times 10^{-2}$ \\
\hline
\end{tabular}

Table 1: Comparison of numerical approximation to the current with the exact and approximate currents given by (2.18) and (2.19) for a range of parameter values.

The meshes produced all cluster the nodes along the electrode surface and close to the boundary singularity at the point $(1,0)$. The part of the final mesh closest to the electrode surface for the problem with $z_{e}=0.5, z_{m}=1$ and $\epsilon_{1}=\epsilon_{2}=0.9214$ is shown in Figure 4. The mesh is coarse and regular further from the electrode surface. Reducing the adaptive tolerance has the effect of refining the mesh further above the electrode surface, but also refining the mesh across the material interfaces as shown in Figure 5.

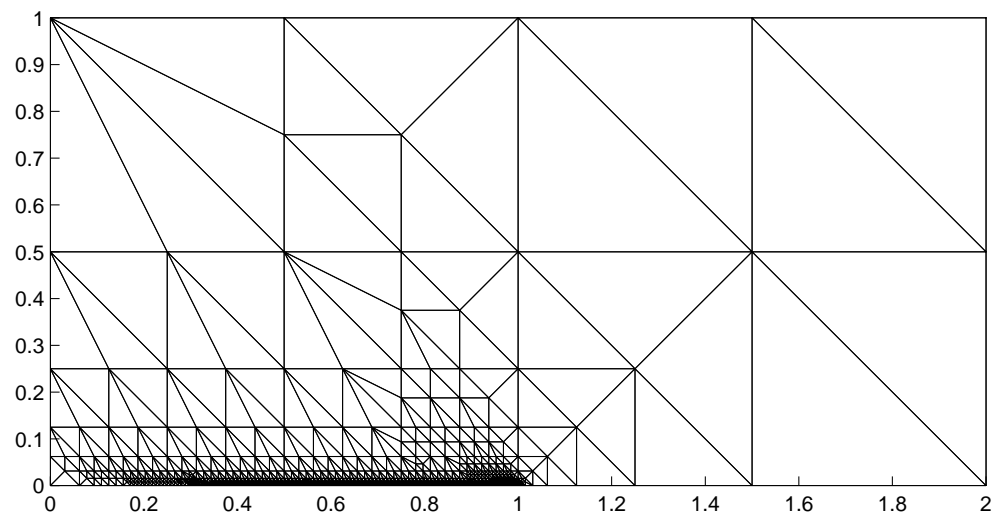

Figure 4: Part of the final mesh produced by the adaptive algorithm.

\section{Discontinuous Finite Element Solution}

We consider shape-regular meshes $\mathcal{T}_{h}=\{\kappa\}$ that partition the domain $\Omega$ into open element domains (squares) $\kappa$. Hanging nodes are allowed, although we shall assume that there is at most one hanging node per element-edge which we assume to be at the centre of the edge. We denote by $h$ the piecewise constant mesh function with $h(x) \equiv h_{\kappa}=\operatorname{diam}(\kappa)$ for $x \in \kappa$. We assume that each element $\kappa \in \mathcal{T}_{h}$ is a smooth 


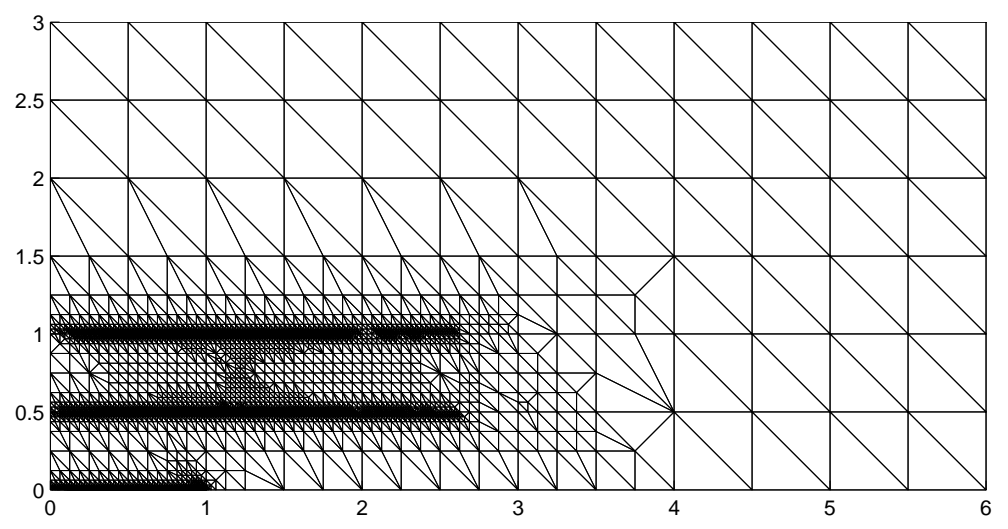

Figure 5: Part of the final mesh produced by the adaptive algorithm with a tolerance of $1 \%$ of the exact current.

bijective image of a fixed reference element $\hat{\kappa}=(-1,1)^{2}$, that is, $\kappa=F_{\kappa}(\hat{\kappa})$ for all $\kappa \in \mathcal{T}_{h}$. On $\hat{\kappa}$ we define $\mathcal{Q}_{p}$ to be the space of polynomials of degree $p \geq 1$ :

$$
\mathcal{Q}_{p}=\operatorname{span}\left\{\hat{x}^{\kappa}: 0 \leq \kappa_{i} \leq p, \quad i=1,2\right\}
$$

To each $\kappa \in \mathcal{T}_{h}$ we assign an integer $p_{\kappa} \geq 1$; collecting the $p_{\kappa}$ and $F_{\kappa}$ in the vectors $\mathbf{p}=\left\{p_{\kappa}: \kappa \in \mathcal{T}_{h}\right\}$ and $\mathbf{F}=\left\{F_{\kappa}: \kappa \in \mathcal{T}_{h}\right\}$, respectively, we introduce the finite element space

$$
S^{\mathbf{p}}\left(\Omega, \mathcal{T}_{h}, \mathbf{F}\right)=\left\{u \in L_{2}(\Omega):\left.u\right|_{\kappa} \circ F_{\kappa} \in \mathcal{Q}_{p_{\kappa}} ; \kappa \in \mathcal{T}_{h}\right\}
$$

An interior edge of $\mathcal{T}_{h}$ is defined as the (non-empty) 1-dimensional interior of $\partial \kappa_{i} \cap$ $\partial \kappa_{j}$, where $\kappa_{i}$ and $\kappa_{j}$ are two adjacent elements of $\mathcal{T}_{h}$, not necessarily matching. A boundary edge of $\mathcal{T}_{h}$ is defined as the (non-empty) 1-dimensional interior of $\partial \kappa \cap \Gamma$, where $\kappa$ is a boundary element of $\mathcal{T}_{h}$. We denote by $\Gamma_{\text {int }}$ the union of all interior edges of $\mathcal{T}_{h}$. Given an edge $e \subset \Gamma_{\text {int }}$, shared by the two elements $\kappa_{i}$ and $\kappa_{j}$, where the indices $i$ and $j$ satisfy $i>j$, we write $\mathbf{n}_{e}$ to denote the (numbering-dependent) unit normal vector which points from $\kappa_{i}$ to $\kappa_{j}$; on boundary faces, we put $\mathbf{n}_{e}=\mathbf{n}$. Further, for $v \in H^{1}\left(\Omega, \mathcal{T}_{h}\right)$ we define the jump of $v$ across $e$ and the mean value of $v$ on $e$, respectively, by

$$
[v]=\left.v\right|_{\partial \kappa_{i} \cap e}-\left.v\right|_{\partial \kappa_{j} \cap e} \quad \text { and } \quad\langle v\rangle=\frac{1}{2}\left(\left.v\right|_{\partial \kappa_{i} \cap e}+\left.v\right|_{\partial \kappa_{j} \cap e}\right)
$$

On a boundary face $e \subset \partial \kappa$, we set $[v]=\left.v\right|_{\partial \kappa \cap e}$ and $\langle v\rangle=\left.v\right|_{\partial \kappa \cap e}$.

\subsection{The $\boldsymbol{h} p$-Discontinuous Galerkin Method}

In order to find the $h p$-DGFEM formulation of the problem we first proceed as in the continuous case; we multiply the governing equation (2.1) by a test function $v$, integrate 
over elements, sum over all elements and apply Green's theorem. This gives

$$
\begin{aligned}
0= & \sum_{\kappa_{e}} \int_{\kappa_{e}}-P_{e} \nabla^{2} p^{\prime} v \mathrm{~d} \Omega+\sum_{\kappa_{m}} \int_{\kappa_{m}}-P_{m} \nabla^{2} p^{\prime} v \mathrm{~d} \Omega+\sum_{\kappa_{s}} \int_{\kappa_{s}}-P_{s} \nabla^{2} p^{\prime} v \mathrm{~d} \Omega \\
= & \sum_{\kappa_{e}} \int_{\kappa_{e}} P_{e} \nabla p^{\prime} \cdot \nabla v \mathrm{~d} \Omega+\sum_{\kappa_{m}} \int_{\kappa_{m}} P_{m} \nabla p^{\prime} \cdot \nabla v \mathrm{~d} \Omega+\sum_{\kappa_{s}} \int_{\kappa_{s}} P_{s} \nabla p^{\prime} \cdot \nabla v \mathrm{~d} \Omega \\
& -\sum_{\kappa_{e}} \int_{\partial \kappa_{e}} P_{e} \frac{\partial p^{\prime}}{\partial n} v \mathrm{~d} s-\sum_{\kappa_{m}} \int_{\partial \kappa_{m}} P_{m} \frac{\partial p^{\prime}}{\partial n} v \mathrm{~d} s-\sum_{\kappa_{s}} \int_{\partial \kappa_{s}} P_{s} \frac{\partial p^{\prime}}{\partial n} v \mathrm{~d} s \\
= & \sum_{\kappa_{e}} \int_{\kappa_{e}} P_{e} \nabla p^{\prime} \cdot \nabla v \mathrm{~d} \Omega+\sum_{\kappa_{m}} \int_{\kappa_{m}} P_{m} \nabla p^{\prime} \cdot \nabla v \mathrm{~d} \Omega+\sum_{\kappa_{s}} \int_{\kappa_{s}} P_{s} \nabla p^{\prime} \cdot \nabla v \mathrm{~d} \Omega \\
& -\int_{\Gamma_{e}} P_{e} \frac{\partial p^{\prime}}{\partial n} v \mathrm{~d} s-\int_{\Gamma_{m}} P_{m} \frac{\partial p^{\prime}}{\partial n} v \mathrm{~d} s-\int_{\Gamma_{s}} P_{s} \frac{\partial p^{\prime}}{\partial n} v \mathrm{~d} s \\
& -\sum_{\kappa_{e}} \int_{\partial \kappa_{e} \backslash \Gamma_{e}} P_{e} \frac{\partial p^{\prime}}{\partial n} v \mathrm{~d} s-\sum_{\kappa_{m}} \int_{\partial \kappa_{m} \backslash \Gamma_{m}} P_{m} \frac{\partial p^{\prime}}{\partial n} v \mathrm{~d} s-\sum_{\kappa_{s}} \int_{\partial \kappa_{s} \backslash \Gamma_{s}} P_{s} \frac{\partial p^{\prime}}{\partial n} v \mathrm{~d} s .
\end{aligned}
$$

For ease of notation we define

$$
\bar{P}= \begin{cases}P_{e} & \text { in } \Omega_{e} \cup\left(\Gamma_{e} \cap \Gamma_{\mathrm{D}}\right), \\ P_{m} & \text { in } \Omega_{m} \cup\left(\Gamma_{m} \cap \Gamma_{\mathrm{D}}\right), \\ P_{s} & \text { in } \Omega_{s} \cup\left(\Gamma_{s} \cap \Gamma_{\mathrm{D}}\right)\end{cases}
$$

We consider the integrals around the boundaries of the three layers:

$$
\begin{aligned}
& \int_{\Gamma_{e}} P_{e} \frac{\partial p^{\prime}}{\partial n} v \mathrm{~d} s+\int_{\Gamma_{m}} P_{m} \frac{\partial p^{\prime}}{\partial n} v \mathrm{~d} s+\int_{\Gamma_{s}} P_{s} \frac{\partial p^{\prime}}{\partial n} v \mathrm{~d} s \\
= & \int_{\Gamma_{e} \cap \Gamma_{\mathrm{D}}} P_{e} \frac{\partial p^{\prime}}{\partial n} v \mathrm{~d} s+\int_{\Gamma_{m} \cap \Gamma_{\mathrm{D}}} P_{m} \frac{\partial p^{\prime}}{\partial n} v \mathrm{~d} s+\int_{\Gamma_{s} \cap \Gamma_{\mathrm{D}}} P_{s} \frac{\partial p^{\prime}}{\partial n} v \mathrm{~d} s \\
& +\int_{\Gamma_{e} \cap \Gamma_{\mathrm{N}}} P_{e} \frac{\partial p^{\prime}}{\partial n} v \mathrm{~d} s+\int_{\Gamma_{m} \cap \Gamma_{\mathrm{N}}} P_{m} \frac{\partial p^{\prime}}{\partial n} v \mathrm{~d} s+\int_{\Gamma_{s} \cap \Gamma_{\mathrm{N}}} P_{s} \frac{\partial p^{\prime}}{\partial n} v \mathrm{~d} s \\
& +\int_{0}^{r_{\max }}-P_{e}\left(\frac{\partial p^{\prime}}{\partial z} v\right)_{z=z_{e}^{-}} r \mathrm{~d} r+\int_{0}^{r_{\max }} P_{m}\left(\frac{\partial p^{\prime}}{\partial z} v\right)_{z=z_{e}^{+}} r \mathrm{~d} r \\
& +\int_{0}^{r_{\max }}-P_{m}\left(\frac{\partial p^{\prime}}{\partial z} v\right)_{z=z_{m}^{-}} r \mathrm{~d} r+\int_{0}^{r_{\max }} P_{s}\left(\frac{\partial p^{\prime}}{\partial z} v\right)_{z=z_{m}^{+}} r \mathrm{~d} r \\
= & \int_{\Gamma_{\mathrm{D}}} \bar{P} \frac{\partial p^{\prime}}{\partial n} v \mathrm{~d} s+\int_{\Gamma_{\mathrm{N}}} \bar{P} \frac{\partial p^{\prime}}{\partial n} v \mathrm{~d} s+\int_{0}^{r_{\max }}\left[\bar{P} \frac{\partial p^{\prime}}{\partial z} v\right]_{z=z_{e}} r \mathrm{~d} r \\
& +\int_{0}^{r_{\max }}\left[\bar{P} \frac{\partial p^{\prime}}{\partial z} v\right]_{z=z_{m}} r \mathrm{~d} r \\
= & \int_{\Gamma_{\mathrm{D}}} \bar{P} \frac{\partial p^{\prime}}{\partial n} v \mathrm{~d} s+\int_{\Gamma_{\mathrm{N}}} \bar{P} \frac{\partial p^{\prime}}{\partial n} v \mathrm{~d} s+\int_{0}^{r_{\max }}\left(\left[\bar{P} \frac{\partial p^{\prime}}{\partial z}\right]\langle v\rangle+[v]\left\langle\bar{P} \frac{\partial p^{\prime}}{\partial z}\right\rangle\right)_{z=z_{e}} r \mathrm{~d} r \\
& +\int_{0}^{r_{\max }}\left(\left[\bar{P} \frac{\partial p^{\prime}}{\partial z}\right]\langle v\rangle+[v]\left\langle\bar{P} \frac{\partial p^{\prime}}{\partial z}\right\rangle\right)_{z=z_{m}} r \mathrm{~d} r .
\end{aligned}
$$


We now use the material interface conditions (2.8) and the fact that $\partial p^{\prime} / \partial n=0$ on $\Gamma_{\mathrm{N}}$. Thus we have

$$
\begin{aligned}
\int_{\Gamma_{e}} P_{e} \frac{\partial p^{\prime}}{\partial n} & v \mathrm{~d} s+\int_{\Gamma_{m}} P_{m} \frac{\partial p^{\prime}}{\partial n} v \mathrm{~d} s+\int_{\Gamma_{s}} P_{s} \frac{\partial p^{\prime}}{\partial n} v \mathrm{~d} s=\int_{\Gamma_{\mathrm{D}}} \bar{P} \frac{\partial p^{\prime}}{\partial n} v \mathrm{~d} s \\
& +\int_{0}^{r_{\max }}\left([v]\left\langle\bar{P} \frac{\partial p^{\prime}}{\partial z}\right\rangle\right)_{z=z_{e}} r \mathrm{~d} r+\int_{0}^{r_{\max }}\left([v]\left\langle\bar{P} \frac{\partial p^{\prime}}{\partial z}\right\rangle\right)_{z=z_{m}} r \mathrm{~d} r .
\end{aligned}
$$

We now substitute (4.7) into (4.4). Adopting the $\bar{P}$ notation throughout, we have

$$
\sum_{\kappa} \int_{\kappa} \bar{P} \nabla p^{\prime} \cdot \nabla v \mathrm{~d} \Omega-\int_{\Gamma_{\mathrm{D}}} \bar{P} \frac{\partial p^{\prime}}{\partial n} v \mathrm{~d} s-\int_{\Gamma_{\mathrm{int}}}\left\langle\bar{P} \nabla p^{\prime} \cdot \mathbf{n}\right\rangle[v] \mathrm{d} s=0 .
$$

The Dirichlet boundary conditions and inter-element continuity across edges in $\Gamma_{\text {int }}$ are imposed in the usual way, so that, on dividing through by $P_{m}$ and setting $\hat{P}=\bar{P} / P_{m}$ we have

$$
\begin{gathered}
\sum_{\kappa} \int_{\kappa} \hat{P} \nabla p^{\prime} \cdot \nabla v \mathrm{~d} \Omega-\int_{\Gamma_{\mathrm{D}}} \hat{P}\left(v \nabla p^{\prime} \cdot \mathbf{n}-\theta p^{\prime} \nabla v \cdot \mathbf{n}\right) \mathrm{d} s+\int_{\Gamma_{\mathrm{D}}} \sigma p^{\prime} v \mathrm{~d} s \\
-\int_{\Gamma_{\mathrm{int}}}\left(\left\langle\hat{P} \nabla p^{\prime} \cdot \mathbf{n}\right\rangle[v]-\theta\langle\hat{P} \nabla v \cdot \mathbf{n}\rangle\left[p^{\prime}\right]\right) \mathrm{d} s+\int_{\Gamma_{\mathrm{int}}} \sigma\left[p^{\prime}\right][v] \mathrm{d} s \\
=\int_{\Gamma_{\mathrm{D}}}(\theta \hat{P} \nabla v \cdot \mathbf{n}+\sigma v) g_{P} \mathrm{~d} s,
\end{gathered}
$$

where $g_{P}$ is the value of $p^{\prime}$ on the Dirichlet boundary

We may now define the $h p$-DGFEM approximation of (2.1) and (2.3)-(2.8) as follows: find $p_{\mathrm{DG}}^{\prime}$ in $S^{\mathbf{p}}\left(\Omega, \mathcal{T}_{h}, \mathbf{F}\right)$ such that

$$
B_{\mathrm{DG}}\left(p_{\mathrm{DG}}^{\prime}, v\right)=\ell_{\mathrm{DG}}(v)
$$

for all $v \in S^{\mathbf{p}}\left(\Omega, \mathcal{T}_{h}, \mathbf{F}\right)$. Here, the bilinear form $B_{\mathrm{DG}}(\cdot, \cdot)$ is defined by

$$
\begin{aligned}
B_{\mathrm{DG}}(w, v)= & \sum_{\kappa \in \mathcal{T}_{h}} \int_{\kappa} \hat{P} \nabla w \cdot \nabla v \mathrm{~d} \Omega+\int_{\Gamma_{\mathrm{int}} \cup \Gamma_{\mathrm{D}}} \sigma\langle\hat{P}\rangle[w][v] \mathrm{d} s \\
& -\int_{\Gamma_{\mathrm{int}} \cup \Gamma_{\mathrm{D}}}\left(\left\langle\hat{P} \nabla w \cdot \mathbf{n}_{e}\right\rangle[v]-\theta\left\langle\hat{P} \nabla v \cdot \mathbf{n}_{e}\right\rangle[w]\right) \mathrm{d} s,
\end{aligned}
$$

and the linear functional $\ell_{\mathrm{DG}}(\cdot)$ is given by

$$
\ell_{\mathrm{DG}}(v)=\int_{\Gamma_{\mathrm{D}}}(\theta \hat{P} \nabla v \cdot \mathbf{n}+\sigma v) g_{P} \mathrm{~d} s .
$$

Here, $\sigma$, the discontinuity-penalisation parameter, is defined by

$$
\left.\sigma\right|_{e}=C_{\sigma}\langle\hat{P}\rangle \frac{\left\langle p^{2}\right\rangle}{\langle h\rangle} \text { for } e \subset \Gamma_{\text {int }} \cup \Gamma_{\mathrm{D}}
$$

where $C_{\sigma}$ is a positive constant, cf. [4].

Selecting the parameter $\theta=-1$ gives rise to the so-called Symmetric Interior Penalty Galerkin (SIPG) scheme which has been shown to give optimal rates of convergence for linear functionals [3]. 


\subsection{Expression for the Current}

We note that the current may be written in the equivalent formulation

$$
I^{\prime}=\int_{\Gamma_{\mathrm{D}}}\left(\theta \nabla p^{\prime} \cdot \mathbf{n}+\sigma p^{\prime} / \bar{P}\right) g_{w} \mathrm{~d} s=J\left(p^{\prime}\right)
$$

where

$$
g_{w}= \begin{cases}0 & \text { on } r_{\max } \text { and } z_{\max } \\ \frac{\pi}{2} & \text { on } z=0,0 \leq r \leq 1\end{cases}
$$

\subsection{An A Posteriori Error Bound}

The a posteriori bound on the error in the computed current is again based on a duality argument. The dual problem is defined to be: find $w \in H^{2}\left(\Omega, \mathcal{T}_{h}\right)$ such that $w$ is continuous and

$$
B_{\mathrm{DG}}(\phi, w)=J(\phi) \quad \forall \phi \in H^{2}\left(\Omega, \mathcal{T}_{h}\right)
$$

We note than when $\theta=-1$ both of the definitions (3.17) and (4.16) give the same strong formulation for the dual problem.

We now recall that the solution to the primal problem satisfies

$$
B_{\mathrm{DG}}\left(p^{\prime}, v\right)=\ell(v) \quad \forall v \in H^{2}\left(\Omega, \mathcal{T}_{h}\right),
$$

and its DGFEM approximation satisfies

$$
B_{\mathrm{DG}}\left(p_{\mathrm{DG}}^{\prime}, v_{h}\right)=\ell\left(v_{h}\right) \quad \forall v_{h} \in S^{\mathbf{p}}\left(\Omega, \mathcal{T}_{h}, \mathbf{F}\right) .
$$

Letting $v=v_{h} \in S^{\mathbf{p}}\left(\Omega, \mathcal{T}_{h}, \mathbf{F}\right)$ in (4.17) and subtracting (4.18) gives the so-called Galerkin orthogonality property

$$
B_{\mathrm{DG}}\left(p^{\prime}-p_{\mathrm{DG}}^{\prime}, v_{h}\right)=0 \quad \forall v_{h} \in S^{\mathbf{p}}\left(\Omega, \mathcal{T}_{h}, \mathbf{F}\right) .
$$

The a posteriori error analysis now proceeds as follows. From the definition of the dual problem (4.16) we have

$$
\begin{aligned}
I^{\prime}-I_{h}^{\prime}=J\left(p^{\prime}\right)-J\left(p_{\mathrm{DG}}^{\prime}\right)=J\left(p^{\prime}-p_{\mathrm{DG}}^{\prime}\right) & =B_{\mathrm{DG}}\left(p^{\prime}-p_{\mathrm{DG}}^{\prime}, w\right) \\
& =B_{\mathrm{DG}}\left(p^{\prime}-p_{\mathrm{DG}}^{\prime}, w-v_{h}\right)
\end{aligned}
$$

for any $v_{h} \in S^{\mathbf{p}}\left(\Omega, \mathcal{T}_{h}, \mathbf{F}\right)$ using the Galerkin orthogonality property (4.19). We now use the bilinearity of $B_{\mathrm{DG}}(\cdot, \cdot)$ to get

$$
\begin{aligned}
I^{\prime}-I_{h}^{\prime} & =B_{\mathrm{DG}}\left(p^{\prime}, w-v_{h}\right)-B_{\mathrm{DG}}\left(p_{\mathrm{DG}}^{\prime}, w-v_{h}\right) \\
& =\ell\left(w-v_{h}\right)-B_{\mathrm{DG}}\left(p_{\mathrm{DG}}^{\prime}, w-v_{h}\right)
\end{aligned}
$$


which is independent of the exact solution $p^{\prime}$. We define the element and boundary residuals

$$
\begin{aligned}
& r_{h, p}^{\kappa}=0-\left(-\hat{P} \nabla^{2} p_{\mathrm{DG}}^{\prime}\right) \quad \text { in element } \kappa \\
& r_{h, p}^{\mathrm{D}}=\left(g_{P}-p_{\mathrm{DG}}^{\prime}\right) \quad \text { on } \Gamma_{\mathrm{D}} \\
& r_{h, p}^{\mathrm{N}}=-\hat{P} \nabla p_{\mathrm{DG}}^{\prime} \cdot \mathbf{n} \quad \text { on } \Gamma_{\mathrm{N}} .
\end{aligned}
$$

Application of Green's theorem and a little algebra then yields the error representation formula

$$
\begin{aligned}
I^{\prime}-I_{\mathrm{DG}}^{\prime}= & \sum_{\kappa} \int_{\kappa} r_{h, p}^{\kappa}\left(w-v_{h}\right) \mathrm{d} \Omega+\int_{\Gamma_{\mathrm{D}}}\left(\theta \hat{P} \nabla\left(w-v_{h}\right) \cdot \mathbf{n}+\sigma\left(w-v_{h}\right)\right) r_{h, p}^{\mathrm{D}} \mathrm{d} s \\
& +\int_{\Gamma_{\mathrm{N}}}\left(w-v_{h}\right) r_{h, p}^{\mathrm{N}} \mathrm{d} s-\int_{\Gamma_{\mathrm{int}}}\left[\hat{P} \nabla p_{\mathrm{DG}}^{\prime} \cdot \mathbf{n}_{e}\right]\left\langle w-v_{h}\right\rangle \mathrm{d} s \\
& -\int_{\Gamma_{\mathrm{int}}}\left(\theta\left\langle\hat{P} \nabla\left(w-v_{h}\right) \cdot \mathbf{n}_{e}\right\rangle+\sigma\left[w-v_{h}\right]\right)\left[p_{\mathrm{DG}}^{\prime}\right] \mathrm{d} s
\end{aligned}
$$

for all $v_{h} \in S^{\mathbf{p}}\left(\Omega, \mathcal{T}_{h}, \mathbf{F}\right)$.

\subsection{Numerical Examples}

We again compare the current values obtained by our $h p$-adaptive discontinuous Galerkin finite element algorithm with those given by the analytical solution (2.18) and the approximate analytical solution (2.19). In each case we have chosen the adaptive tolerance to be the same as that used in the case of continuous piecewise linear basis functions. The background meshes also consist of the same set of nodes. However, we find that for our discontinuous Galerkin method the adaptive algorithm converges with just one iteration (i.e. no mesh refinement or polynomial enrichment is needed to achieve the tolerance). The results are shown in Table 2 and again we see good agreement in all cases. In some cases the current values obtained are significantly more accurate than those using the continuous adaptive algorithm.

\begin{tabular}{lllcccc}
\hline$z_{e}$ & $z_{m}$ & $\epsilon_{1}=\epsilon_{2}$ & Eq. (2.18) & Eq. (2.19) & $I_{h}^{\prime}$ & error bound \\
\hline 0.5 & 1 & 0.9214 & 0.5141 & 0.5236 & 0.5171 & $1.1536 \times 10^{-2}$ \\
0.5 & 1 & 0.1 & 0.9665 & 0.9663 & 0.9851 & $3.0128 \times 10^{-2}$ \\
2 & 4 & 0.9765 & 0.6672 & 0.7116 & 0.6752 & $1.4347 \times 10^{-2}$ \\
2 & 4 & 0.9214 & 0.7530 & 0.7605 & 0.7567 & $1.7933 \times 10^{-2}$ \\
2 & 4 & 0.2090 & 0.9683 & 0.9681 & 0.9734 & $2.8832 \times 10^{-2}$ \\
2 & 2.5 & 0.9 & 0.8667 & 0.8667 & 0.8707 & $2.3524 \times 10^{-2}$ \\
2 & 2.5 & 0.1 & 0.9942 & 0.9941 & 0.9995 & $3.0249 \times 10^{-2}$ \\
\hline
\end{tabular}

Table 2: Comparison of numerical approximation to the current using DGFEM with the exact and approximate currents given by (2.18) and (2.19) for a range of parameter values. 
In order to see the adaptive algorithm at work, we consider the problem with $z_{e}=0.5$, $z_{m}=1$ and $\epsilon_{1}=\epsilon_{2}=0.9214$ and we set the tolerance to be $0.1 \%$ of the exact current. Part of the final mesh is shown in Figure 6; beyond the illustrated part of the domain the mesh is coarse and regular and the polynomial degree is one. We see that the mesh concentrates extra nodes around the boundary singularity at $(1,0)$ where the electrode meets the insulator. There are also extra nodes at the material interfaces. Where refinement is needed away from these singularities it takes the form of $p$-refinement although the maximum polynomial degree does not exceed four. The whole mesh has a total of 1981 elements and 8836 degrees of freedom. This is a considerable improvement on the adaptive algorithm with continuous piecewise linear basis functions which required 34811 degrees of freedom to solve the problem with the same parameters and an adaptive tolerance 10 times larger. If we use an adaptive tolerance of $1 \%$ of the exact current (as in the adaptive algorithm with continuous piecewise linear basis functions) we require only 6996 degrees of freedom.
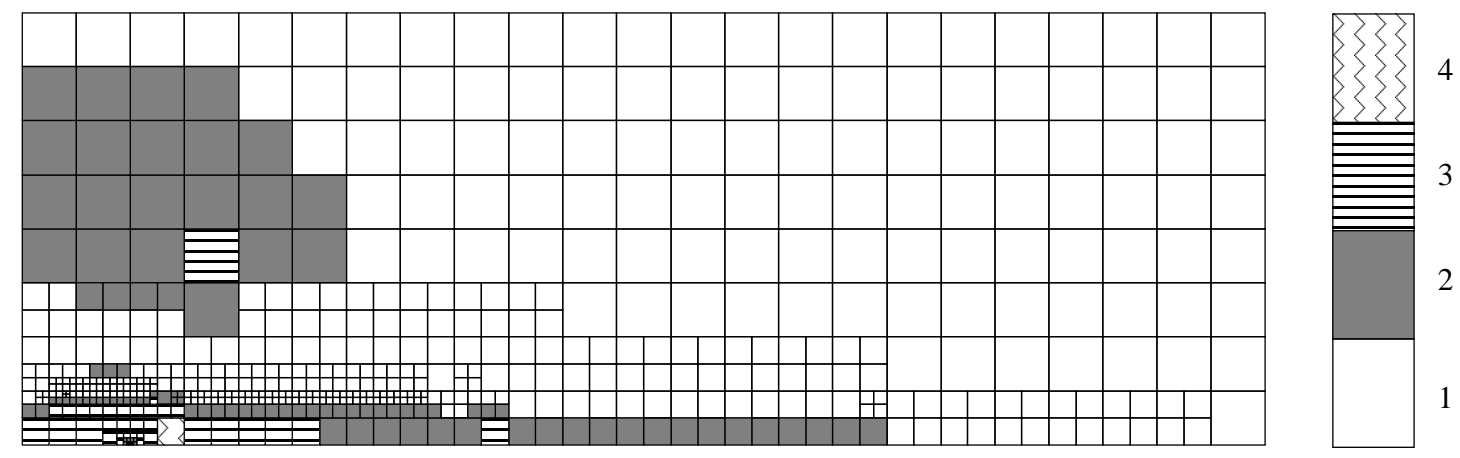

Figure 6: Part of the final mesh produced by the adaptive algorithm.

\section{Conclusions}

We have presented two adaptive finite element algorithms for the solution of the shielded microdisc electrode problem. The first algorithm was based on the use of continuous piecewise linear finite element methods and performed only $h$-refinement whilst the second was an $h p$-DGFEM. We derived a posteriori bounds on the error in the computed current in both cases; these bounds formed the basis of the mesh refinement algorithm. We found that for a range of experimental parameters both algorithms were able to compute the current to within the specified tolerance using relatively coarse meshes although our discontinuous Galerkin method was more efficient, achieving the prescribed tolerance on the first mesh, suggesting that this should be the method of choice for the solution of such problems. 


\section{Acknowledgements}

The authors are pleased to acknowledge the financial support of the EPSRC (Grant Number: GR/R17041).

\section{References}

[1] L.C. Clark, 1956. Monitor and control of blood and tissue oxygen tensions. Transactions of the American Society for Internal Organs, 2: 41-46.

[2] J. Galceran, J. Salvador, J. Puy, J. Cecilia and D.J. Gavaghan, 1997. Analytical solution for the steady-state diffusion towards an inlaid disc microelectrode in a multi-layered medium. J. Electroanal. Chem., 440(1-2): 1.

[3] K. Harriman, P. Houston, W. Senior and E. Süli, 2003. hp-Version Discontiuous Galerkin Finite Element Methods with Interior Penalty for Partial Differential Equations with Nonnegative Characteristic Form. In C.-W. Shu, T. Tang and S.-Y. Cheng, editors, Recent Advances in Scientific Computing and Partial Differential Equations, Volume 330 of Contemporary Mathematics, page 89. AMS, Providence, RI.

[4] P. Houston, Ch. Schwab and E. Süli, 2002. Discontinuous $h p$-finite element methods for advection-diffusion-reaction problems. SIAM J. Numer. Anal., 39(6): 2133. 